\title{
MCM-BP regulates unloading of the MCM2-7 helicase in late S phase
}

\author{
Atsuya Nishiyama, ${ }^{1}$ Lori Frappier, ${ }^{2}$ and Marcel Méchali ${ }^{1,3}$ \\ ${ }^{1}$ Institute of Human Genetics, CNRS, 34396 Montpellier, France; ${ }^{2}$ Department of Molecular Genetics, University of Toronto, \\ Toronto, Ontario M5S 1A8, Canada
}

\begin{abstract}
Origins of DNA replication are licensed by recruiting MCM2-7 to assemble the prereplicative complex (pre-RC). How MCM2-7 is inactivated or removed from chromatin at the end of $S$ phase is still unclear. Here, we show that MCM-BP can disassemble the MCM2-7 complex and might function as an unloader of MCM2-7 from chromatin. In Xenopus egg extracts, MCM-BP exists in a stable complex with MCM7, but is not associated with the MCM2-7 hexameric complex. MCM-BP accumulates in nuclei in late $S$ phase, well after the loading of MCM2-7 onto chromatin. MCM-BP immunodepletion in Xenopus egg extracts inhibits replication-dependent MCM dissociation without affecting pre-RC formation and DNA replication. When excess MCM-BP is incubated with Xenopus egg extracts or immunopurified MCM2-7, it binds to MCM proteins and promotes disassembly of the MCM2-7 complex. Recombinant MCM-BP also releases MCM2-7 from isolated late-S-phase chromatin, but this activity is abolished when DNA replication is blocked. MCM-BP silencing in human cells also delays MCM dissociation in late S phase. We propose that MCM-BP plays a key role in the mechanism by which pre-RC is cleared from replicated DNA in vertebrate cells.
\end{abstract}

[Keywords: MCM2-7; MCM-BP; DNA replication; chromatin; Xenopus; cell cycle]

Supplemental material is available for this article.

Received June 7, 2010; revised version accepted November 23, 2010.

To maintain genome integrity, origins of DNA replication must be activated once and only once per cell cycle. Origins are licensed for replication by the formation of the prereplicative complex (pre-RC). Pre-RC assembly requires loading of the MCM2-7 complex onto DNA through the coordinated action of origin recognition complex (ORC), Cdc6, and Cdt1, and is regulated by Geminin and MCM9 (Remus and Diffley 2009). Once licensing has occurred, MCM2-7 complexes are tightly associated with chromatin, and ORC, Cdc6, and Cdt1 become dispensable to hold MCM2-7 on DNA (Bochman and Schwacha 2009). The MCM2-7 complex serves as a platform to recruit Cdc45 and GINS, thus converting the pre-RC into a preinitiation complex (pre-IC). Cdc45 and GINS then induce the helicase activity of the MCM2-7 complex, promoting DNA unwinding at origins of DNA replication (for review, see Remus and Diffley 2009).

The MCM2-7 proteins belong to the $\mathrm{AAA}^{+}$superfamily of ATPases and predominantly exist as an heterohexameric complex of $\sim 600 \mathrm{kDa}$ (Forsburg 2004). In addition to such hexameric complexes, MCM subcomplexes (MCM4/6/7, MCM2/4/6/7, and MCM3/5) have also been

${ }^{3}$ Corresponding author.

E-MAIL mechali@igh.cnrs.fr; FAX 33-499-619-920.

Article published online ahead of print. Article and publication date are online at http://www.genesdev.org/cgi/doi/10.1101/gad.614411. reported (Prokhorova and Blow 2000). Although their biological significance is still unclear, MCM4/6/7 is thought to possess core ATPase and DNA helicase activity, whereas MCM2/3/5 provides regulatory roles (Ishimi 1997; Schwacha and Bell 2001). Structural analysis showed that MCM2-7 form a double hexameric ring with head-to-head configuration, and DNA passes through the central channel of the double hexamer (Fletcher et al. 2003; Evrin et al. 2009; Remus et al. 2009). Although it is possible that MCM2-7 oligomerization occurs after its chromatin binding (Maiorano et al. 2000), it is likely that toroid opening is necessary for MCM2-7 unloading from chromatin.

MCM2-7 is displaced from replicated DNA during S phase and cannot be reloaded at origins during $S$ phase, ensuring that each of the many origins can be activated only once during each cell cycle. A number of mechanisms that negatively regulate pre-RC assembly have been identified (Arias and Walter 2007). In metazoans, reloading of MCM2-7 in S phase is prevented by downregulation of Cdt1 activity in two ways. First, Cdt1 is inactivated by Geminin after activation of the replication origins. Second, Cdt1 is destroyed by ubiquitin-mediated proteolysis in a manner that depends on the initiation of DNA replication. In addition, several inhibitory mechanisms involving Orc1 and Cdc6 have been reported (Delmolino et al. 2001; Mendez et al. 2002). However, 
ORC, Cdc6, and Cdt1 are dispensable for retaining MCM2-7 on chromatin. It is therefore likely that there are additional mechanisms to inactivate the MCM2-7 helicase following S-phase progression.

MCM-BP was identified as a novel MCM-binding protein by tandem affinity purification of MCM proteins from human cells (Sakwe et al. 2007). It is highly conserved among higher eukaryotes. Human MCM-BP can associate with MCM3-7 but not MCM2, and hexameric MCM2-7 complexes lack MCM-BP. A fraction of human MCM-BP binds to chromatin in a cell cycle-dependent manner like other MCM proteins, and silencing of $M C M-B P$ by siRNA reduced the amount of MCM4 on chromatin, but cell growth was not significantly impaired (Sakwe et al. 2007). Finally, a mutant of plant MCM-BP has been reported to display G2 cell cycle arrest (Takahashi et al. 2008, 2010). Thus, MCM-BP may have an important role in S-phase progression, but its function, like its effect on the MCM complex, remains unclear.

Here, we characterized the function of MCM-BP using in vitro systems derived from Xenopus egg extracts. We find that MCM-BP can disassemble the MCM2-7 hexameric complex and functions as an unloader of MCM2-7 from chromatin after DNA replication. We also show that MCM-BP has a similar function in human cells. Our results show that MCM2-7 hexamer rings can be opened by MCM-BP to promote MCM2-7 dissociation at the end of the replicon synthesis.

\section{Results}

Xenopus MCM-BP binds to MCM7 but is not part of the MCM2-7 complex

To characterize the functional properties of MCM-BP in Xenopus egg extracts, we prepared and purified rabbit polyclonal antibodies against $\mathrm{His}^{10}$-tagged, full-length recombinant Xenopus MCM-BP, a 626-amino-acid-long protein that is $72 \%$ identical to human MCM-BP (Supplemental Fig. S1). This antibody, but not the preimmune serum, specifically recognized a protein with an apparent molecular weight of $70 \mathrm{kDa}$ in Xenopus egg extracts (Fig. $1 \mathrm{~A}$ ) and also in vitro translated Xenopus MCM-BP (Supplemental Fig. S2A). By comparing the signal in egg extracts with given amounts of recombinant MCM-BP run in parallel, we estimated the concentration of MCM-BP in Xenopus egg extracts to be $10 \mathrm{ng} / \mu \mathrm{L}$ or $140 \mathrm{nM}$ /data not shown). In Xenopus oocytes, which were arrested at the prophase stage of the first meiotic division, MCM-BP was localized in the nucleus (GV), like MCM proteins (Supplemental Fig. S2B).

To confirm that Xenopus MCM-BP is a MCM-binding protein, and to determine its relationship with the MCM2-7 complex, Xenopus interphase egg extracts were immunoprecipitated with polyclonal anti-MCM-BP and anti-MCM7 antibodies. The anti-MCM7 antibody coimmunoprecipitated other MCM subunits, as expected, as well as a large amount of MCM-BP (Fig. 1B; Supplemental Fig. S2C). The anti-MCM-BP antibody coprecipitated MCM7 as well as MCM3 and MCM5, but not MCM2,
A

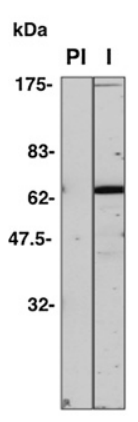

B

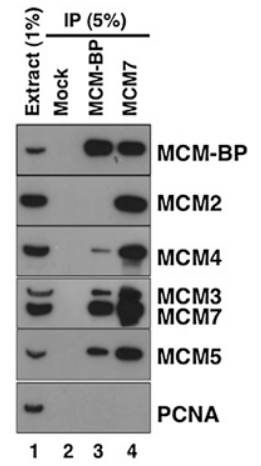

C

\begin{tabular}{|c|c|c|c|c|}
\hline \multirow{2}{*}{} & \multicolumn{4}{|c|}{ IP } \\
\cline { 2 - 5 } & \multicolumn{2}{|c|}{ MCM-BP } & \multicolumn{2}{c|}{ MCM3 } \\
\cline { 2 - 5 } & Score & $\begin{array}{c}\text { Peptide } \\
\text { numbers }\end{array}$ & Score & $\begin{array}{c}\text { Peptide } \\
\text { numbers }\end{array}$ \\
\hline MCM2 & - & - & 3032 & 40 \\
\hline MCM3 & 1192 & 26 & 3546 & 36 \\
\hline MCM4 & 479 & 11 & 2619 & 36 \\
\hline MCM5 & 1355 & 28 & 1625 & 31 \\
\hline MCM6 & 463 & 9 & 1086 & 23 \\
\hline MCM7 & 1355 & 31 & 1650 & 31 \\
\hline
\end{tabular}

Figure 1. Xenopus MCM-BP binds to MCM proteins. $(A)$ Western blot analysis of $0.5 \mu \mathrm{L}$ of Xenopus interphase egg extracts with the rabbit polyclonal anti-MCM-BP antibody (I) or preimmune serum (PI). (B) MCM-BP associates with MCM proteins in Xenopus interphase egg extracts. Immunoprecipitations carried out using anti-MCM-BP (lane 3) or anti-MCM7 (lane 4) antibodies were analyzed by Western blotting using the indicated antibodies. Also shown is a mock immunoprecipitation to determine background signals (lane 2) and the proteins present in untreated egg extracts $(0.5 \mu \mathrm{L})$ (lane 1). (C) MCM-BP and MCM3 immunoprecipitates from Xenopus interphase egg extracts were analyzed by MS. MS profiles were identified using the Mascot search engine. Score: Mascot scores.

and very little MCM4 (Fig. 1B). These data are in agreement with observations in human cells (Sakwe et al. 2007). Mass spectrometry (MS) analysis of the MCM-BP and MCM3 immunoprecipitates confirmed these results (Fig. 1C). Altogether, these data suggest that MCM-BP associates with MCM proteins, but it is not a cofactor of the whole MCM2-7 complex.

To further investigate the composition of the different MCM subcomplexes, Xenopus interphase egg extracts were fractionated by sucrose gradient sedimentation. The MCM2-7 complex sedimented as a major $600-\mathrm{kDa}$ complex, and a second complex of $\sim 200 \mathrm{kDa}$ that contained mainly MCM7 was also observed (Fig. 2A). The abundance of this second complex varied with the extracts, as observed previously (Coue et al. 1998; Prokhorova and Blow 2000). Interestingly, this second complex was more abundant in extracts in which DNA replication was finished than in extracts treated with Geminin (Supplemental Fig. $\mathrm{S} 3 \mathrm{~A})$, suggesting that the formation of this subcomplex is actively regulated during DNA replication. MCM-BP was not present in the 600-kDa MCM2-7 complex that contains the full MCM2-7 hexamer (Fig. 2A). However, MCM$\mathrm{BP}$ did not sediment at a position corresponding to its 
A

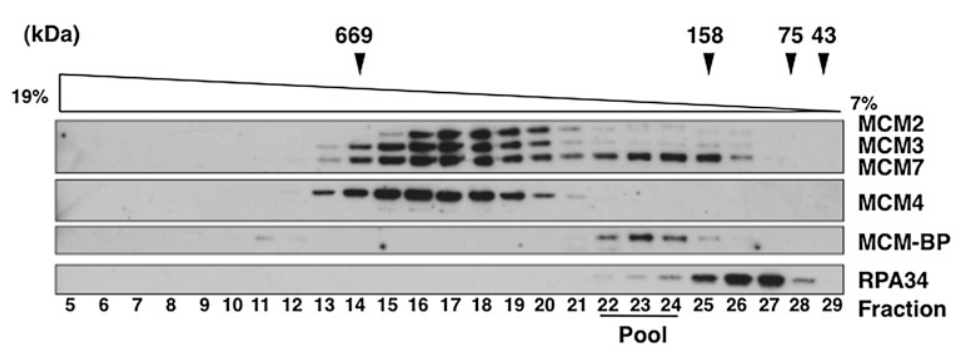

B
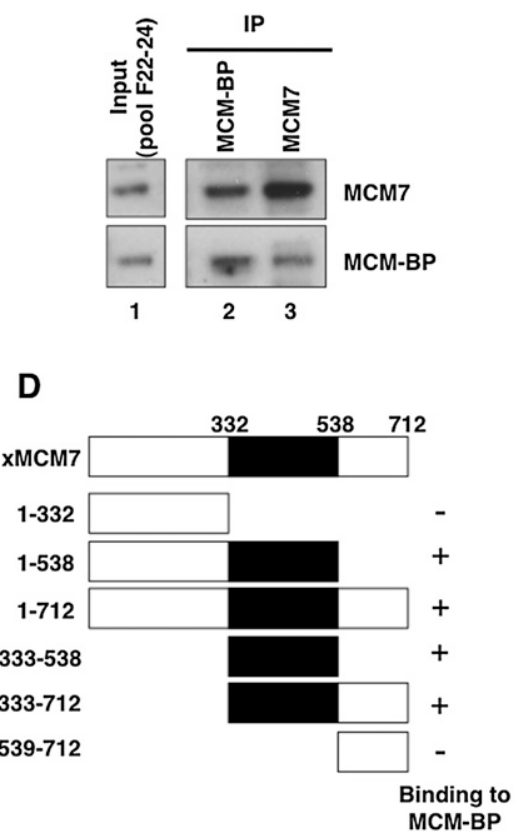

C

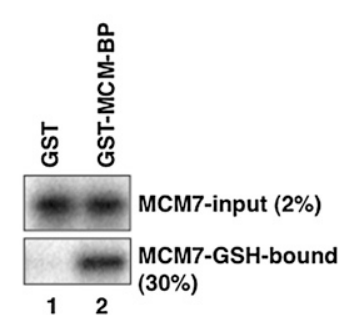

Figure 2. MCM-BP forms subcomplexes with MCMs, mainly with MCM7. (A) Western blot analysis of Xenopus interphase egg extracts following fractionation on a linear 7\%-19\% sucrose gradient (see the Materials and Methods). (B) Low-molecular-weight fractions from the sucrose gradient shown in $A$ (fractions 22-24) were immunoprecipitated using anti-MCM-BP (lane 2) and anti-MCM7 (lane 3) antibodies as indicated. Immunoprecipitated proteins were analyzed by Western blotting using antibodies against MCM7 and MCM-BP. Proteins present in the pool were also analyzed. $(C) \mathrm{MCM} 7$ was radiolabeled with ${ }^{35} \mathrm{~S}$-methionine during in vitro translation in a reticulocyte lysate system and then incubated with GST control (lane 1) or GST-MCM-BP (lane 2). GST complexes were isolated with GSH-Sepharose beads and analyzed by autoradiography. Two percent of the reaction was analyzed as input. (D) MCM-BP binds to the conserved MCM box in MCM7. (Left) Schematic representation of MCM7 deletion mutants. GST (lanes 1-6) or GST-MCM-BP (lanes 7-12) was bound to GSHSepharose beads and incubated with either Myc-tagged recombinant full-length MCM7 or the various deletion mutants. (Right) Coprecipitated proteins were analyzed by Western blotting using the anti-Myc antibody.

molecular weight $(70 \mathrm{kDa})$, but was detected as a 150 - to 200-kDa protein complex, in agreement with the presence of a MCM-BP-MCM7 complex (70 kDa + $81 \mathrm{kDa})$. Immunoprecipitation of pooled fractions that corresponded to the MCM-BP peak confirmed that MCM-BP was associated with MCM7 (Fig. 2B). MCM3 or MCM4 was hardly coimmunoprecipitated with MCM-BP after the sedimentation (data not shown), probably due to the dissociation of the complex during the long centrifugation $(22 \mathrm{~h})$. Indeed, the MCM-BP-MCM7 complex was rather stable, as it was resistant to high-salt elution $(0.8 \mathrm{M} \mathrm{NaCl})$ (Supplemental Fig. S3B), whereas MCM2 and MCM4 were mostly eluted in the high-salt fraction (Supplemental Fig. S3B; data not shown). The MCM7 (or MCM4)MCM-BP complex could be detected in both interphase and mitotic extracts, suggesting that its formation is not cell cycle-dependent (Supplemental Fig. S3C).

The MCM-BP-MCM7 interaction was confirmed by GST pull-down experiments, showing that the two proteins interact with each other (Fig. 2C). To identify what part of MCM7 was involved in the association with MCM-BP, in vitro translated Myc-tagged wild-type MCM7 and deletion mutants (Supplemental Fig. S4A) were incubated with
GST-MCM-BP or GST, and coprecipitates were analyzed by Western blotting. We found that the MCM7 deletion mutants, which lack the conserved MCM box that includes the canonical Walker A and B motifs, could not bind to MCM-BP (Fig. 2D, lanes 7,12). Consistent with this, MCM-BP could bind efficiently to a fragment of MCM7 that contained only this domain (Fig. 2D, lane 10). These experiments indicate that the interaction between MCM-BP and MCM7 relies on the MCM box of MCM7. We also found that a lysine-to-alanine point mutation within the conserved Walker A motif of MCM7 did not impair this interaction (Supplemental Fig. S4B). Given that the lysine residue in the Walker A motif is essential for nucleotide-binding and DNA-unwinding activities (You et al. 2002), this result suggests that ATP or its hydrolysis is not necessary for MCM7 to be associated with MCM-BP.

$M C M-B P$ is imported in the nucleus in late $S$ phase, when MCM2-7 dissociates from chromatin

The association of MCM-BP with MCM7, a subunit of the MCM2-7 helicase, led us to ask whether MCM-BP could play a role during DNA replication. Upon addition 
of sperm nuclei to Xenopus interphase egg extracts, pre-RC formation on chromatin occurred within $10 \mathrm{~min}$ through binding of ORC2, MCM3, and MCM7 (Fig. 3A). Nuclear membrane formation around the chromatin occurred within $20 \mathrm{~min}$ (data not shown), allowing initiation of replication and binding of Replication Protein A (RPA) to chromatin (Fig. 3A,B), as expected. RPA, MCM3, and MCM7 were then released from chromatin during the ongoing DNA synthesis (Fig. 3A, right panel). MCM-BP was detected in the nucleus only by mid-S phase (Fig. 3A, left panel). However, despite its interaction with MCM7, MCM-BP was not stably bound to chromatin (Fig. 3A, right panel). Moreover, MCM-BP nuclear import strikingly correlated with MCM2-7 dissociation from chromatin and with the progressive increase of the MCM7-MCM-BP association (Fig. 3C). These data suggest that MCM-BP is involved in the final stages of DNA replication rather than during initiation of DNA synthesis or DNA elongation.

\section{MCM-BP is involved in the dissociation of MCM2-7 at the end of $S$ phase}

To further define the role of MCM-BP during DNA replication, Xenopus interphase egg extracts were immunodepleted of MCM-BP using the affinity-purified polyclonal anti-MCM-BP antibody (Fig. 3D; Supplemental Fig. S5), and their replication profile was further analyzed. MCMBP depletion did not significantly affect the total MCM2-7 content of the extract, in agreement with the large excess

A

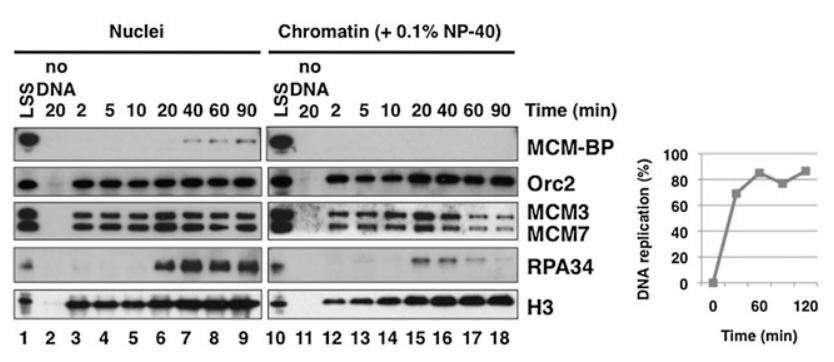

of MCM2-7 complex in the extract (Fig. 3D). Recruitment of ORC2, MCM3, and MCM7 to chromatin was not significantly affected under these conditions (Fig. 3E). Similar experiments using membrane-free egg extracts-which assemble pre-RCs, including MCM2-7, but cannot activate DNA synthesis (Coue et al. 1998; Mimura and Takisawa 1998) — confirmed that MCM-BP depletion did not impair pre-RC assembly, judging from normal Orc2, Cdt1, and MCM2-7 loading onto chromatin (Supplemental Fig. S6). In complete extracts, loading onto chromatin of Cdc45 (a pre-IC component) (data not shown) and of PCNA (an elongation complex factor) (Fig. 3D) was also normal in MCM-BP-depleted extracts, and DNA replication was achieved with kinetics comparable with that of mockdepleted extracts (Fig. 3F). Conversely, under this condition, depletion of MCM-BP significantly affected MCM2-7 dissociation from chromatin during DNA synthesis (Fig. 3E; Supplemental Fig. S7A), in agreement with the finding that MCM-BP is imported into the nucleus only from mid-S phase (Fig. 3A). We found that the timing and dissociation of the MCM2-7 complex were reproducibly delayed when MCM-BP was depleted (Supplemental Fig. S7A), but not irreversibly blocked, probably due to

Figure 3. MCM-BP accumulates in the nucleus in late $S$ phase and is not required for pre-RC formation and DNA replication, but is involved in MCM2-7 dissociation. (A) Time course of MCM-BP accumulation in the nucleus (left panel) and binding to chromatin (right panel). Sperm nuclei (3000 per microliter) were added to Xenopus interphase egg extracts, and then intact nuclei (lanes 3-9) and detergent-resistant chromatin fractions (lanes 12-18) were isolated at the indicated time points during DNA replication (see the Materials and Methods) and analyzed by Western blotting. A sample of egg extracts $(0.5 \mu \mathrm{L}$ ) (lanes 1,10) (LSS, low-speed supernatant) or insoluble material obtained by centrifugation of egg extracts (lanes 2,11) (no DNA) was also included as control. (B) Replication efficiency of the reaction described in $A$. $(C)$ Time course of MCM-BP binding to MCM7. Xenopus sperm nuclei (XSP) were added to Xenopus interphase egg extracts, and intact nuclei were isolated and separated into chromatin and soluble nuclear fractions at different time points during DNA replication, as described in the Materials and Methods. (Top panel) MCM2-7 unloading from chromatin was assessed using anti-MCM3 and anti-MCM7 antibodies. (Bottom panels) Soluble nuclear fractions were immunoprecipitated with anti-MCM7 antibodies, and immunoprecipitates were analyzed by Western blotting with antiMCM3, anti-MCM7, and anti-MCM-BP antibodies. $(D)$ One microliter of mock- and MCM-BP-depleted (using the rabbit polyclonal anti-MCM-BP antibody) interphase egg extracts was analyzed by Western blotting with the indicated antibodies to confirm MCM-BP depletion. (E) MCM-BP regulates MCM2-7 unloading from chromatin during late $S$ phase. Xenopus interphase egg extracts were immunodepleted with IgG fractions purified from preimmune serum (left panels) or with the rabbit polyclonal anti-MCM-BP antibody (right panels). Sperm nuclei (3000 per microliter) were added to Xenopus egg extracts, and chromatin fractions were isolated at the indicated times during DNA replication (see the Materials and Methods). Chromatinbound proteins were analyzed by Western blotting using the indicated antibodies. $(F)$ Replication efficiency of the reaction described in $E$. 
alternative mechanisms that can clear the MCM2-7 after DNA replication (see the Discussion). We then investigated whether depletion of MCM-BP would inhibit replicon endjoining by analysis of replication products using alkaline gel electrophoresis. Replicating DNA isolated from MCMBP-depleted extracts progressively accumulated highmolecular-weight DNA chains similarly to mock-depleted extracts, indicating that replicated DNA from adjacent replicons could converge normally and join in the absence of MCM-BP (Supplemental Fig. S7C). We conclude that MCM-BP depletion inhibits and delays MCM2-7 dissociation during $\mathrm{S}$ phase by a mechanism that does not involve replicon end-joining.

A similar inhibition of DNA synthesis-dependent removal of MCMs was observed in the presence of aphidicolin, an inhibitor of DNA polymerase $\alpha$ (Fig. 4A, cf. lanes 1 and 2). However, inhibition of MCM2-7 unloading by aphidicolin was accompanied by Chk1 phosphorylation (Fig. 4A, lanes 2,4) and accumulation of RPA onto chromatin (Zou and Elledge 2003; Byun et al. 2005; data not shown). Conversely, MCM-BP depletion did not induce checkpoint activation at 120 min after sperm addition, as judged by the lack of Chk1 phosphorylation and RPA accumulation (Fig. 4A, lane 3; see also Supplemental Fig. S8B; data not shown). Thus, the presence of chromatinbound MCM2-7 alone is not sufficient to induce checkpoint response.

We conclude that DNA synthesis is necessary for MCM2-7 dissociation from chromatin, but that intra-S checkpoint activation is unlikely to be the mechanism whereby MCM dissociation is inhibited upon MCM-BP depletion. These data also confirm that MCM-BP is involved in a late step of DNA replication during MCM2-7 unloading from chromatin.

In order to substantiate the specificity of the effect observed in MCM-BP-depleted extracts, we tried to rescue MCM2-7 unloading in these extracts by adding $15 \mathrm{ng} / \mu \mathrm{L}$ recombinant MCM-BP, a concentration comparable with that of endogenous MCM-BP (10-20 ng/ $\mu \mathrm{L}$ ) (Fig. 4B). Recombinant MCM-BP clearly rescued MCM3 and MCM7 unloading in the late stage of $S$ phase (Fig. 4C, cf. lanes 4 and 2; see also Supplemental Fig. S8A,C). In addition, MCM-BP released MCM2-7 from chromatin only at the end of $S$ phase, but not during pre-RC or pre-IC formation. Indeed, incubation with recombinant MCM-BP had no effect on MCM2-7 in pre-RCs assembled in high-speed extracts (Fig. 4D), in which pre-RCs are efficiently formed but DNA replication cannot initiate, as described above.

We then investigated whether MCM-BP could accelerate MCM2-7 dissociation from chromatin and at what time during $S$ phase. Chromatin was isolated at various times during $S$ phase and was further incubated with recombinant MCM-BP. MCM proteins were efficiently dissociated from chromatin only when MCM-BP was added after $45 \mathrm{~min}$ (Fig. 4E), but not during pre-RC formation. In agreement with this result and with our observations using membrane-free egg extracts (Fig. 4D), recombinant MCMBP could not release MCM2-7 from chromatin when initiation of DNA synthesis was blocked by p21 (Fig. 4E). Thus, p21 inhibits MCM2-7 removal, confirming that
A

C

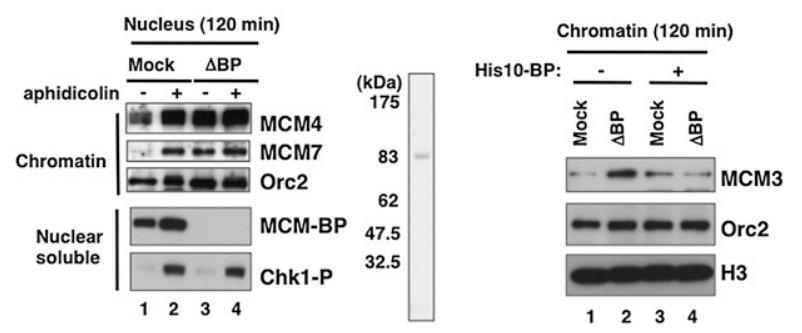

D

E
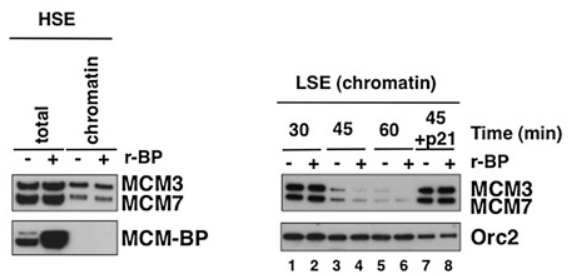

Figure 4. Efficient MCM2-7 dissociation from chromatin involves MCM-BP and S-phase progression. (A) MCM-BP depletion does not cause checkpoint activation. Sperm nuclei $(6000$ per microliter) were added to mock- or MCM-BP-depleted $(\triangle \mathrm{BP})$ Xenopus interphase egg extracts in the absence or presence of 50 $\mu \mathrm{g} / \mathrm{mL}$ aphidicolin. Nuclei were isolated $120 \mathrm{~min}$ after sperm addition, and both chromatin-bound fractions (top panels) and soluble nuclear fractions (bottom panels) were analyzed by Western blotting using the indicated antibodies. $(B)$ Purification of recombinant MCM-BP. His-tagged full-length MCM-BP was expressed and further purified by nickel chromatography. The purified protein was analyzed by SDS-PAGE followed by staining with Coomassie blue. (C) Purified recombinant MCM-BP rescues MCM3 unloading from chromatin in MCM-BP-depleted Xenopus egg extracts. Egg extracts were mock-depleted with IgG fractions purified from preimmune serum (lanes 1,3) or immunodepleted with the rabbit polyclonal anti-MCM-BP antibody (lanes 2,4). Then, buffer alone (lanes 1,2) or $0.2 \mu \mathrm{M}$ recombinant MCM-BP (rBP) (lanes 3,4) was added to the depleted extracts before sperm addition (3000 per microliter). Onehundred-twenty minutes later, chromatin fractions were isolated and analyzed by Western blotting for MCM3, ORC2, and H3. $(D, E)$ Recombinant MCM-BP releases MCM2-7 from chromatin only in late $S$ phase. Chromatin or nuclei were isolated from high-speed egg extracts (HSE) $(D)$ or low-speed egg extracts (LSE) (E) treated with buffer or recombinant p21, and incubated with $3 \mu \mathrm{M}$ recombinant MCM-BP $(\mathrm{rBP})$ in CPB buffer containing $0.2 \%$ Triton X-100. After $20 \mathrm{~min}$ of incubation at room temperature, soluble supernatants were removed by centrifugation, and chromatin fractions were analyzed by Western blotting using the indicated antibodies.

DNA synthesis is necessary for further MCM2-7 displacement from chromatin (Fig. 4E, cf. lanes 3,4 and 7,8) and recombinant MCM-BP cannot displace MCM2-7 under these conditions (Fig. 4E, cf. lanes 7 and 8). Similar results were obtained following aphidicolin treatment (data not shown).

Altogether, these results suggest that MCM-BP regulates the dissociation of MCM2-7 from chromatin after ongoing DNA synthesis, and that, during pre-RC or preIC assembly, MCM binding to chromatin is not affected. 
We then wished to address the mechanism by which MCM2-7 is unloaded from chromatin by MCM-BP. Here we showed that MCM-BP binds to MCM7 but is not detected in the whole MCM2-7 complex. One explanation could be that, when MCM-BP binds to the MCM2-7 complex, the complex is simultaneously dissociated, resulting in its release from chromatin. To test this hypothesis, recombinant MCM-BP was added to Xenopus egg extracts and the composition of the MCM2-7 complex was analyzed by sucrose gradient density centrifugation. Recombinant MCM-BP induced a dramatic change in the sedimentation profile of the complex, as a large part of the MCM2-7 complex was dissociated in lowmolecular-weight fractions (Fig. 5A). To identify the resulting complexes, pooled high- or low-molecular-weight fractions ( $\mathrm{H}$ and $\mathrm{L}$ fractions in Fig. 5A) were immunoprecipitated with anti-MCM7 antibodies. High-molecularweight fractions from control and extracts with excess MCM-BP (Fig. 5B, " $\mathrm{H}^{\prime \prime}$ ) contained the MCM7 subunit but no MCM-BP (see also Fig. 2A). In low-molecular-weight fractions from control extracts (Fig. 5B, " $\mathrm{L}^{\prime}$ ), MCM7 was associated with MCM-BP and also with MCM3 and MCM4.
Conversely, in low-molecular-weight fractions from extracts with excess MCM-BP, only association of MCM7 with MCM-BP could be detected, but not with MCM2, MCM3, or MCM4 (Fig. 5B, cf. lanes 2 and 4). MCM3 immunoprecipitation showed that the association of MCM3 with MCM2 and MCM4 was also lost (Supplemental Fig. S9). These results imply that binding of MCMBP to MCM7 not only releases MCM7 from the whole MCM2-7 complex, but also destabilizes the interactions of each individual subunit. We also noticed that endogenous MCM-BP, in the complex with MCM7, was replaced by recombinant MCM-BP under this condition, suggesting that the binding of MCM-BP to MCM7 is dynamic.

Then, we asked whether MCM-BP could directly dissociate the isolated MCM2-7 complex. To this aim, immunopurified MCM2-7 complex (see the Materials and Methods) was incubated with recombinant MCM-BP or Geminin, an inhibitor of MCM loading to chromatin, as a control. After incubation with MCM-BP, a substantial part of the MCM2-7 complex was dissociated, releasing MCM subunits in the eluate (Fig. 5C). Dissociation did not occur upon incubation with buffer alone, purified
A

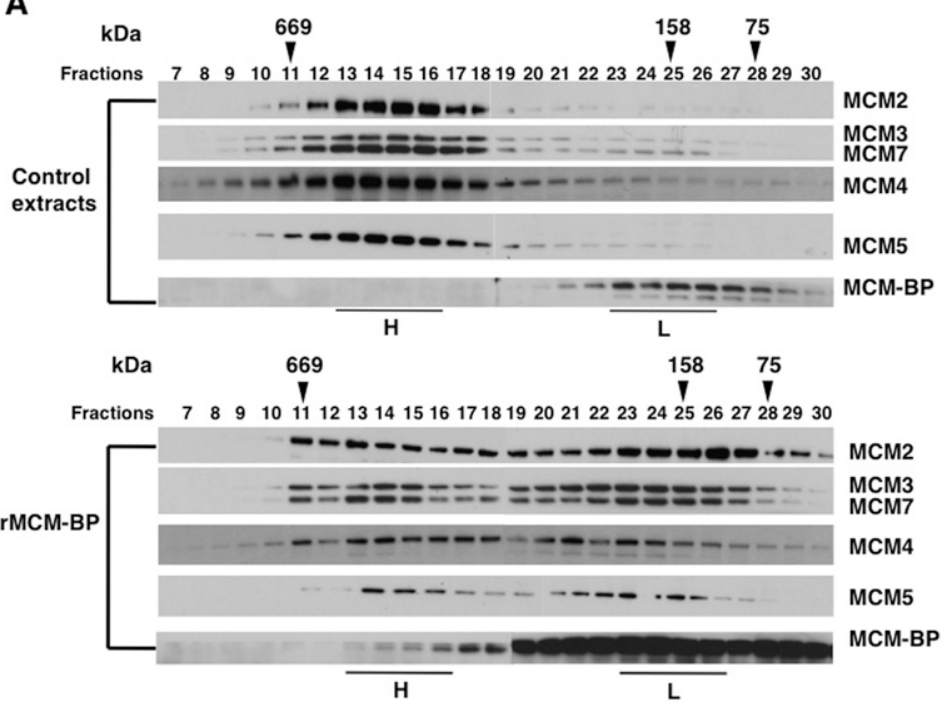

B

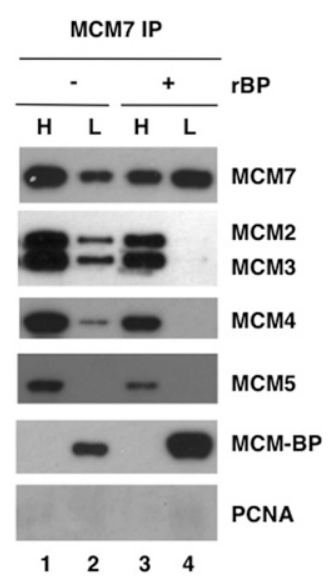

C

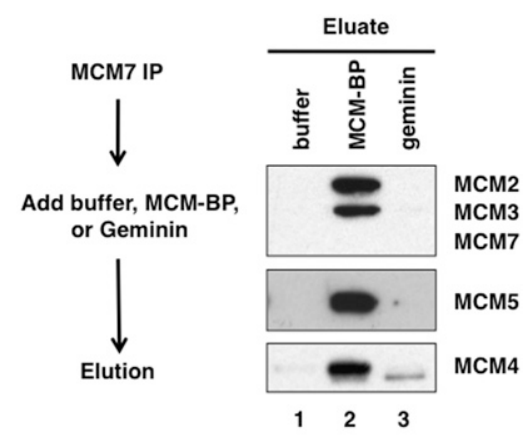

Figure 5. Excess MCM-BP dissociates the MCM2-7 hexameric complex. (A) Xenopus interphase egg extracts were incubated or not with $2 \mu \mathrm{M}$ recombinant MCM-BP (rBP) for $1 \mathrm{~h}$ without sperm addition. They were then fractionated on a linear $7 \%-19 \%$ sucrose gradient. The indicated proteins were detected by Western blot analysis. (B) Pooled high-molecularweight fractions (fractions 13-16; H) and low-molecular-weight fractions (fractions 23-26; L) were immunoprecipitated with anti-MCM7 antibodies (lanes 1-4) and analyzed by Western blotting. $(C)$ The MCM2-7 complex was immunoprecipitated from Xenopus interphase egg extracts with anti-MCM7 antibodies. MCM7 precipitates were washed and incubated with purified recombinant MCM-BP $(2 \mu \mathrm{M})$ (lane 2) or Geminin (2 $\mu \mathrm{M}$ ) (lane 3) for $1 \mathrm{~h}$. The proteins released in the eluate were analyzed by Western blotting with antibodies against different MCM proteins. 
recombinant Geminin, or MCM2 (Fig. 5C; data not shown). Importantly, this reaction was not affected by either the absence of ATP or the presence of ATP analogs like ATP- $\gamma$ S (Supplemental Fig. S10) and AMP-ANP (data not shown). From this set of experiments, we conclude that MCM-BP can dissociate the MCM2-7 complex in a reaction that does not require ATP.

Finally, we investigated the consequences of silencing $M C M-B P$ in HeLa cells by using siRNAs. In HeLa cells, most of MCM-BP was observed only in the chromatin-free fraction supernatants after cell fractionation (Fig. 6A; Materials and Methods) and did not cosediment with the MCM2-7 complex in glycerol density gradient fractionation (Fig. 6B) like in Xenopus egg extracts. siRNA efficiently depleted MCM-BP from HeLa cells (Fig. 6A, cf. lanes 1 and 2), and we examined DNA replication by monitoring incorporation of BrdU into the cells. In comparison with

A B
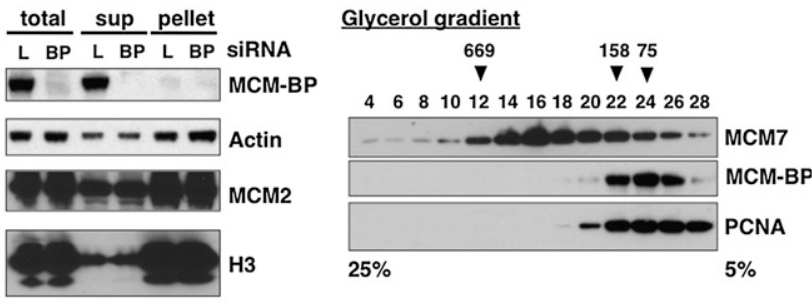

C

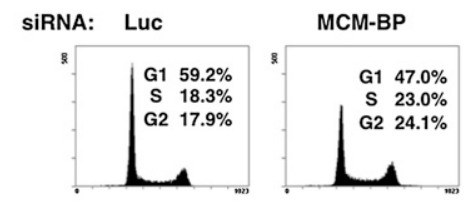

D

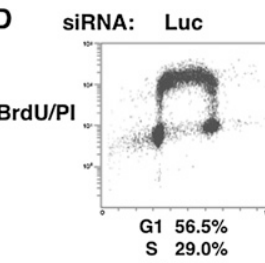

G2 $29.0 \%$
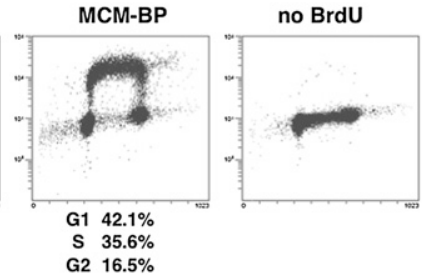

Figure 6. $M C M-B P$ silencing in HeLa cells increases the G2/M population. (A) Human HeLa cells were transfected with Luciferase siRNA (lanes 1,3,5) or MCM-BP siRNA (lanes 2,4,6). Cells were fractionated (see the Materials and Methods) and immunoblotted to assess the expression of MCM-BP, Actin, MCM2, and H3. (B) After CSK extraction, soluble HeLa supernatant was loaded onto a linear $5 \%-25 \%$ glycerol gradient and centrifuged at 26,000 rpm in an SW55Ti rotor for $22 \mathrm{~h}$. Fractions were subjected to SDS-PAGE, and the indicated proteins were detected by Western blot analysis. $(C, D)$ Forty-eight hours after two rounds of transfection with Luciferase or MCM-BP siRNAs, HeLa cells were incubated or not with BrdU for 15 min and stained with propidium iodide, and their DNA content was analyzed by flow cytometry. $(C)$ The DNA content is shown on the $X$-axis, and the relative number of cells is indicated on the $Y$-axis. $(D)$ The intensity of BrdU incorporation is indicated on the logarithmic $Y$-axis; the DNA content is shown on the $X$-axis. cells transfected with control siRNA, cells transfected with $M C M-B P$ siRNA showed a slight increase in the number of cells in $S$ and G2/M phase of the cell cycle but similar BrdU incorporation (Fig. 6C,D). To directly assess the effect of $M C M-B P$ silencing on cell cycle progression, cells were synchronized at the G1/S boundary by doublethymidine block after two rounds of siRNA transfection. Cells were then released from the block, and cell lysates were prepared at different time points and analyzed by immunoblotting. Concomitantly, we evaluated cell cycle progression by FACS analysis. More than $80 \%$ MCM-BP was depleted after two rounds of transfection with siRNA SMART pools specific for MCM-BP (Fig. 7A). FACS analysis again showed that $S$-phase progression was not significantly affected by MCM-BP depletion (Fig. 7B). However, consistent with the Xenopus data, dissociation of MCM7 from chromatin fractions was delayed compared with control cells. We also observed that entry into mitosis occurred more slowly in the absence of MCM-BP, as judged by the delayed accumulation of phosphorylation of $\mathrm{H} 3$ at Ser 10 (Fig. 7A) in comparison with control cells. Altogether, our data suggest that, in human cells, MCM-BP regulates proper G2/M progression by controlling MCM2-7 dissociation from chromatin rather than being involved in bulk DNA replication.

\section{Discussion}

Clearing the replication complex from chromatin is essential before mitosis, and several mechanisms are involved in this function. The findings described in this study suggest that MCM-BP contributes specifically to the dissociation of the MCM2-7 ring complex.

MCM-BP is highly conserved among higher eukaryotes from fission yeast to mammals, but we were unable to detect a MCM-BP ortholog in Saccharomyces cerevisiae. However, in this organism, MCM2-7 is inactivated by nuclear export in a cdk-dependent manner (Nguyen et al. 2001). In other organisms, including fission yeast, MCM2-7 is constitutively localized in the nucleus (Yanow et al. 2001), and therefore additional mechanisms to inhibit MCM2-7 would be required. In fission yeast, global analyses showed that the MCM-BP homolog localizes in the nucleus and appears essential for viability (Matsuyama et al. 2006; Kim et al. 2010), suggesting a crucial role during the cell cycle. This hypothesis is supported by the finding that plant cells with a MCM-BP mutant have a defect in G2/M cell cycle progression. Our results show that, in Xenopus egg extracts, MCM-BP is involved in the release of the MCM2-7 complex from chromosomes during the late stage of $\mathrm{S}$ phase, in agreement with the observations in other organisms. Moreover, in human cells, where the first MCM-BP gene was identified, we detected very little association of MCM-BP with chromatin, and MCM-BP silencing induced delayed MCM7 dissociation from chromatin. Although in our experiments we used detergent concentrations that, in the Xenopus system, are known to entirely remove the nuclear membrane but leave on chromatin all the proteins involved in DNA replication, we cannot rule out the possibility that a minor fraction of MCM-BP might 
A

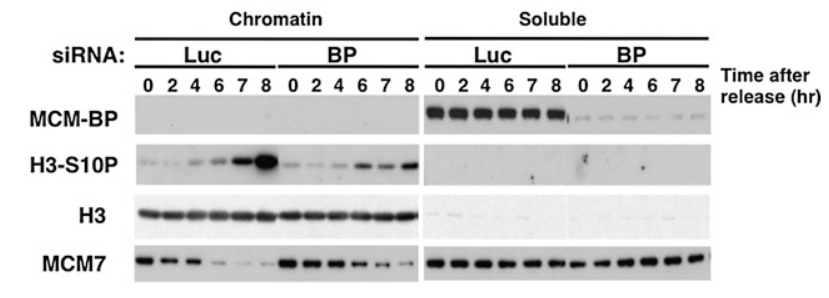

B

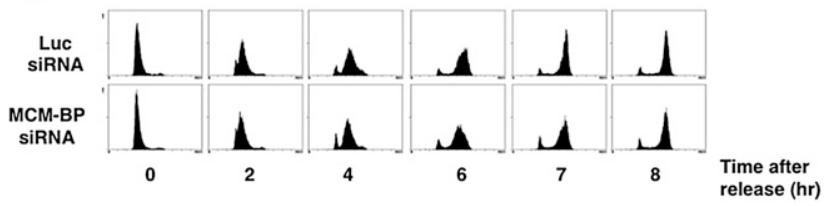

Figure 7. $M C M-B P$ silencing in HeLa cells results in defective mitotic entry. HeLa cells were synchronized by double-thymidine block and release. Cells transfected with Luciferase or $M C M-B P$ siRNAs were incubated with $2 \mathrm{mM}$ thymidine for 15 $\mathrm{h}$ followed by washing with prewarmed PBS and medium. Cells were released into fresh medium for $9 \mathrm{~h}$ and then blocked again with $2 \mathrm{mM}$ thymidine for $17 \mathrm{~h}$. Cells were washed with prewarmed PBS and medium before release into fresh medium. Cells were processed for immunoblotting $(A)$ and FACS analysis $(B)$ at the indicated time points after synchronization.

bind to chromatin in human cells in less stringent conditions. Interestingly, Sakwe et al. (2007) showed that MCMBP stayed longer on chromatin in late $S$ to G2 phase in human cells, when MCM2-7 was almost displaced from DNA, in agreement with the idea that MCM-BP functions as an unloader of MCM2-7. As reported previously in human cells, we found that MCM-BP-silenced cells continued to grow and incorporate BrdU, suggesting that the defect in these cells was not directly due to impaired DNA replication.

\section{$M C M-B P$ and the regulation of unloading} of the MCM2-7 ring complex

Dissociation of MCM2-7 from chromatin at the end of S phase is important to prevent rereplication of DNA and to ensure genome duplication only once per cell cycle. In this study, we provide evidence that MCM-BP is involved in DNA replication-dependent MCM2-7 unloading from chromatin in Xenopus interphase egg extracts. After loading onto chromatin during pre-RC formation, MCM2-7 tightly associates with chromatin, and progression of DNA replication is necessary for its dissociation. We also demonstrate that MCM-BP regulates the dissociation of the MCM2-7 complex by physically interacting mainly with its MCM7 subunit. Our results suggest that MCM-BP is transiently recruited to chromatin to release MCM2-7 from DNA at the end of the synthesis of replicons. This is in agreement with the observation that MCM-BP enters the nucleus during S-phase progression, and that its nuclear import kinetics correlates with MCM2-7 release from chromatin. In Xenopus, replicons are short, with DNA replication origins spaced every 10-20 kb (Hyrien and Mechali 1993;
Walter and Newport 1997). The pre-RC formation extends for $\sim 20 \mathrm{~min}$, after which the replication forks proceed at a speed estimated to be $0.6 \mathrm{~kb} / \mathrm{min}$ (Blow et al. 2001; Lemaitre et al. 2005). The first replicons to be activated will therefore reach their termination point after 35-45 min. This is the time when MCM-BP is imported into the nucleus and the MCM2-7 complex starts to be released from chromatin. Since MCM-BP binding to the MCM2-7 complex simultaneously promotes its dissociation from chromatin, chromatin-bound MCM-BP is unlikely to be detected during this reaction. A possible dynamic interaction of MCM-BP with MCM7 could also be part of a resetting mechanism, which would allow the unloaded MCM2-7 to be used in subsequent cell cycles.

Several observations, including those here, indicate that DNA replication has to be induced to allow MCM2-7 dissociation from chromatin. MCM2-7 dissociation is abolished when initiation of DNA synthesis is inhibited in aphidicolin-treated extracts, despite the presence of nuclear MCM-BP. Similarly, recombinant MCM-BP cannot dissociate MCM2-7 from chromatin that was isolated early in S phase, including licensed chromatin in membrane-depleted high-speed egg extracts (Fig. 4D,E). We think that the stable association of MCM2-7 with the pre-RC or pre-IC complex might prevent MCM-BP from functioning at this stage. MCM2-7 dissociation by MCMBP might therefore require the MCM2-7 helicase to have left the replication origins and traveled along the DNA. An additional possibility could be that MCM-BP is required for inactivation of the MCM2-7 complex in the nucleoplasm to prevent its reassociation with chromatin at the end of $S$ phase.

Altogether, our data suggest that MCM-BP controls the opening of the MCM2-7 complex at the end of S phase through its interaction with MCM7. Interestingly, the MCM7/4 site is the active site involved in DNA unwinding by the MCM2-7 complex (Kanter et al. 2008; Bochman and Schwacha 2009), suggesting that disruption of this site will not only dissociate the MCM complex, but also inactivate it.

\section{Implications of MCM2-7 clearing from chromatin at the end of $S$ phase}

The release of the MCM2-7 complex from chromatin might be crucial at three points: twice during $S$ phase (at the end of replication of replicons and to remove the MCM complexes loaded at silenced origins) and once at mitosis. Here we show that MCM-BP depletion does not alter DNA synthesis or affect Chk1 phosphorylation, suggesting that loss of MCM-BP is not sufficient for inducing rereplication. In metazoans, Geminin synthesis and Cdt1 degradation are independent and redundant mechanisms to prevent rereplication. We cannot exclude the possibility that MCM-BPdependent removal of MCM2-7 from chromatin provides additional safeguards against rereplication.

Before chromosome segregation in mitosis, DNA-bound components during $\mathrm{S}$ phase might become obstacles to the formation of metaphase chromosomes and/or chromosome segregation (Yanagida 2009). Therefore, these components 
might have to be removed. Failure to do so may result in defects in the mitotic chromosomes' assembly (Cuvier et al. 2006, 2008). The MCM2-7 complex tightly binds in excess to interphase chromatin (resistant to high-salt treatment), and its removal from chromatin is therefore critical for progression into mitosis. We found that silencing of $M C M-B P$ in human cells also resulted in defects in mitotic entry (Fig. 7) similar to what was observed in plant cells (Takahashi et al. 2010). In addition to the role of mitotic cyclin-dependent kinases in cleaning replicated chromosomes for mitosis, MCM-BP also could take part in opening and dissociating the MCM2-7 ring complex from chromatin. MCM-BP absence is not sufficient to completely block MCM2-7 dissociation from chromatin, and cells may have evolved different but redundant mechanisms to ensure the removal of pre-RC. At least two mechanisms might contribute to the complete dissociation of MCM2-7 from replicated chromatin. First, a high concentration of recombinant CDK2-Cyclin E negatively regulates pre-RC assembly (Hua et al. 1997) and accelerates the interphase/mitosis transition (Moore et al. 2002), while a comparable concentration of S-CDK in nucleoplasmic extracts does not block Cdt1-dependent MCM2-7 loading and DNA replication (Arias and Walter 2005). Enrichment of Cdk2-Cyclin E in the nucleus may contribute to some late processes in the cell cycle, including MCM2-7 dissociation. Second, the Topoisomerase II-dependent pathway, which is responsible for removing RPA and ORC reservoirs from chromatin (Cuvier et al. 2008), could also play a role in MCM2-7 clearing after replication. Indeed, we observed that ICRF-193, an inhibitor of Topoisomerase II, could also suppress the dissociation of MCM2-7 in late S phase (data not shown). These mechanisms would ensure that the removal of pre-RC occurs before mitosis, thus eliminating the obstacles for mitotic chromosome assembly.

\section{Materials and methods}

Xenopus oocytes, eggs, and egg extracts

Interphasic low-speed supernatants and high-speed supernatants were prepared as described (Maiorano et al. 2005; Lutzmann and Mechali 2008). Low-speed supernatants were finally clarified by centrifugation at 20,000 rpm in an SW55Ti rotor for $20 \mathrm{~min}$. Western blot analyses of oocytes were performed as described (Lemaitre et al. 2002).

\section{Plasmids and proteins}

Full-length Xenopus MCM-BP and MCM7 cDNAs were purchased from Imagene. Xenopus MCM2 cDNA was provided by D. Maiorano. Full-length MCM-BP and MCM2 cDNA and the cDNA fragment of MCM7 that encodes amino acids 545-720 were cloned into pET16b to be expressed as His-tagged proteins. Full-length MCM-BP was also subcloned in the pET24-GST-TEV vector (GST-tagged MCM-BP). His- and GST-tagged fusion proteins were expressed in Escherichia coli BL21 codon plus RIL cells (Stratagene) and were purified as described (Lutzmann et al. 2006). Purified proteins were loaded onto PD-10 desalting columns equilibrated with XB buffer $(10 \mathrm{mM} \mathrm{HEPES} / \mathrm{KOH}$ at $\mathrm{pH} 7.7,100 \mathrm{mM} \mathrm{KCl}, 0.1 \mathrm{mM} \mathrm{CaCl}_{2}, 1 \mathrm{mM} \mathrm{MgCl}_{2}, 2 \%$ sucrose) containing $1 \mathrm{mM}$ DTT, and then concentrated with Vivaspin (Millipore). His-tagged Geminin was purified as described (Lutzmann et al. 2006). Translation in reticulocyte lysates was performed according to the manufacturer's instructions (TNT Reticulocyte Lysates System, Promega). For translation, MCM$\mathrm{BP}$ and MCM7 cDNAs were cloned into the pcDNA3 vector. The MCM7 mutant in which Lys 386 was substituted by alanine was generated by PCR-mediated mutagenesis.

\section{Antibodies}

Anti-Xenopus MCM-BP antisera were obtained by immunizing rabbits with recombinant, full-length His10-tagged MCM-BP; expressed in E. coli; and purified by SDS-PAGE. Antisera were further affinity-purified with the recombinant protein immobilized onto a nitrocellulose membrane. Anti-Xenopus MCM7 antibodies were raised as described above, by immunizing rabbits with a recombinant His10-tagged MCM7 fragment (545-720). Anti-human MCM2 and MCM5 rabbit antibodies that cross-react with Xenopus MCM2 and MCM5 and anti-human MCM7 mouse monoclonal antibody that also recognizes Xenopus MCM7 were purchased from Abcam. A mouse monoclonal antibody against PCNA and a polyclonal anti-histone $\mathrm{H} 3$ antibody were from Sigma and Abcam, respectively. The anti-Phospho-Chk1 and anti-Phospho-Myc antibodies were from Cell Signaling. The antibody against Xenopus Cdc45 was kindly provided by J. Walter. Antibodies against Xenopus Orc2, MCM3, Cdt1, and RPA, and human MCM-BP were described previously (Maiorano et al. 2005; Sakwe et al. 2007; Lutzmann and Mechali 2008).

\section{Nuclei and chromatin isolation from Xenopus egg extracts}

Nuclei and chromatin fractions were prepared from Xenopus egg extracts incubated with 3000-6000 demembraned sperm nuclei per microliter. At the end of the incubation period, the reaction was diluted with 4 vol of $\mathrm{CPB}\left(50 \mathrm{mM} \mathrm{KCl}, 5 \mathrm{mM} \mathrm{MgCl}_{2}, 20 \mathrm{mM}\right.$ $\mathrm{HEPES} / \mathrm{KOH}$ at $\mathrm{pH} 7.7,2 \%$ sucrose), and nuclei were purified by centrifugation at $6000 \mathrm{~g}$ through a sucrose cushion containing $0.7 \mathrm{M}$ sucrose dissolved in CPB for $5 \mathrm{~min}$. For chromatin purification, nuclei pellets were solubilized in $40 \mu \mathrm{L}$ of CPB containing $0.1 \%$ NP-40. Chromatin fractions were collected by centrifugation at $10,000 \mathrm{~g}$ for $5 \mathrm{~min}$. The final supernatant was used as nuclear lysates for immunoprecipitations that were performed with antibodies bound to protein A-agarose (Roche).

\section{Immunoprecipitations}

Five micrograms of the antibody for immunoprecipitation was incubated with protein A-agarose beads for $2 \mathrm{~h}$ at room temperature and washed in $\mathrm{XB}$ buffer. Xenopus egg extracts were fivefold diluted in $\mathrm{XB}$ and incubated with the antibody-bound beads for $1 \mathrm{~h}$ at $4^{\circ} \mathrm{C}$. Beads were then washed with XB twice, with XB containing $0.1 \%$ Triton X-100 once, and with XB alone twice. Proteins were finally eluted with SDS sample buffer and analyzed by SDS-PAGE.

\section{Sucrose and glycerol gradients}

Sucrose or glycerol density gradient centrifugation was performed as described (Lutzmann and Mechali 2008). Briefly, egg extracts were diluted fivefold in XB and centrifuged at 12,000 $\mathrm{g}$ for $10 \mathrm{~min}$ at $4^{\circ} \mathrm{C}$. Cleared egg extract or human cell lysate $(200 \mu \mathrm{L})$ was layered onto a linear gradient prepared in XB supplemented with protease inhibitors. The gradient was then centrifuged at $26,000 \mathrm{~g}$ for $22 \mathrm{~h}$ at $4^{\circ} \mathrm{C}$ in a SW 55Ti rotor, and fractions were collected. 


\section{In vitro dissociation of the MCM2-7 complex}

To follow the disassembly of MCM2-7 complex by MCM-BP, Xenopus interphase egg extracts were incubated with $2 \mu \mathrm{M}$ recombinant MCM-BP or Geminin for $30 \mathrm{~min}$ at room temperature. Egg extracts were cleared by centrifugation and analyzed by sucrose density gradient centrifugation as described above. When sperm chromatin was added to extract, dilution of extracts was performed in XB containing $0.2 \%$ Triton X-100 and centrifuged to remove insoluble materials from egg extracts. For immunopurification of the MCM2-7 complex, purified rabbit polyclonal antiMCM7 antibodies or control IgGs were coupled to protein A-agarose beads, incubated with diluted Xenopus egg extracts for $1 \mathrm{~h}$ at $4^{\circ} \mathrm{C}$, and then washed as described above. Proteins specifically bound to the beads were incubated with $2 \mu \mathrm{M}$ MCM-BP or Geminin in $50 \mu \mathrm{L}$ of XB supplemented with an ATP-regenerating system $(10 \mathrm{mM}$ creatine phosphate, $10 \mu \mathrm{g} / \mathrm{mL}$ creatine kinase, $1 \mathrm{mM}$ ATP, $1 \mathrm{mM} \mathrm{MgCl} 2$ ) for $30 \mathrm{~min}$ at room temperature. To see the effect of recombinant MCM-BP to the MCM2-7 on chromatin, intact nuclei were isolated from egg extracts as described above. Then, nuclei pellets were incubated for $15 \mathrm{~min}$ at room temperature in $40 \mu \mathrm{L}$ of $\mathrm{CPB}$ containing $0.2 \%$ Triton X-100 and an ATP-regenerating system with or without recombinant MCM-BP at a concentration of $3 \mu \mathrm{M}$. Chromatin fractions were collected by centrifugation at 10,000 $\mathrm{g}$ for $5 \mathrm{~min}$ and washed once in XB. Chromatin-bound proteins were analyzed by SDS-PAGE and Western blotting.

\section{Human tissue culture cells}

Asynchronous HeLa cells were grown in Dulbecco's modified Eagle's medium containing $10 \%$ fetal calf serum. SMART pool siRNAs specific for MCM-BP (J-014474-09, J-014474-10, and J-014474-11) and control siRNA (SMART pool nontargeting pool) were obtained from DharmaFect. The siRNAs (33 nM) were transfected into HeLa cells with Lipofectamine2000 according to the manufacturer's instructions (Invitrogen). Cells were subcultured for $48 \mathrm{~h}$ after two rounds of transfection with siRNA. Subsequently, cells were labeled with $10 \mu \mathrm{g} / \mathrm{mL}$ BrdU for $15 \mathrm{~min}$. Cells were fixed with $70 \%$ ethanol, incubated with $20 \mu \mathrm{g} / \mathrm{mL}$ RNase A for $1 \mathrm{~h}$, and treated with $2 \mathrm{~N} \mathrm{HCl}$ for $30 \mathrm{~min}$. Cells were incubated with anti-BrdU antibodies for $1 \mathrm{~h}$ and then stained with fluorescent-conjugated secondary antibodies and propidium iodide according to standard procedures. FACS analysis was performed with a FACSCalibur flow cytometer (BD Biosciences).

\section{Isolation of chromatin fractions from synchronized human cells}

HeLa cells were synchronized at the G1/S boundary by doublethymidine block with $2 \mathrm{mM}$ thymidine. Cells were harvested; suspended in CSK buffer (10 mM PIPES-KOH at pH 6.8, $100 \mathrm{mM}$ $\mathrm{NaCl}, 300 \mathrm{mM}$ sucrose, $1.5 \mathrm{mM} \mathrm{MgCl}_{2}, 0.1 \%$ Triton X-100) containing $5 \mathrm{mM}$ EDTA, $1 \mathrm{mM}$ DTT, $0.1 \mathrm{mM}$ sodium vanadate, $1 \mathrm{mM} \mathrm{NaF}, 10 \mathrm{mM} \beta$-glycerophosphate, $0.1 \mu \mathrm{M}$ tautomycin, and $0.5 \mathrm{mM}$ PMSF; and lysed for $10 \mathrm{~min}$ on ice. Chromatin-enriched fractions were collected by centrifugation at $1300 \mathrm{~g}$ for $3 \mathrm{~min}$ and washed in CSK buffer. For fractionation by sucrose or glycerol density gradient, soluble supernatants were further clarified by centrifugation at $12,000 \mathrm{~g}$ for $10 \mathrm{~min}$.

\section{Acknowledgments}

We thank J. Walter for MCM7 and Cdc45 antibodies; F. Ishikawa for recombinant p21; P. Pasero, D. Maiorano, and M. Lutzmann for critical reading of this manuscript; $\mathrm{S}$. Bocquet for antibody production; M. Nakamura for assistance with cultured cells; and L. Gonzalez, M. Lutzmann, P. Coulombe, O. Ganier, S. Traver, N. Montel, and other members of the Mechali laboratory for sharing unpublished results, experimental assistance, and many helpful discussions. This work is supported by the CNRS, the Ligue contre le Cancer, the National Agency for Research, and the ERC, and is partly supported by grant number 84306 from the Canadian Institutes of Health Research to L.F. L.F. is a tier 1 Canada Research Chair in Molecular Virology, and therefore receives salary support from the Canada Research Chairs program. A.N. is supported by the Association pour la Recherche sur le Cancer and the Uehara Foundation.

\section{References}

Arias EE, Walter JC. 2005. Replication-dependent destruction of Cdt 1 limits DNA replication to a single round per cell cycle in Xenopus egg extracts. Genes Dev 19: 114-126.

Arias EE, Walter JC. 2007. Strength in numbers: Preventing rereplication via multiple mechanisms in eukaryotic cells. Genes Dev 21: 497-518.

Blow JJ, Gillespie PJ, Francis D, Jackson DA. 2001. Replication origins in Xenopus egg extract are 5-15 kilobases apart and are activated in clusters that fire at different times. I Cell Biol 152: 15-25.

Bochman ML, Schwacha A. 2009. The Mcm complex: Unwinding the mechanism of a replicative helicase. Microbiol Mol Biol Rev 73: 652-683.

Byun TS, Pacek M, Yee MC, Walter JC, Cimprich KA. 2005. Functional uncoupling of MCM helicase and DNA polymerase activities activates the ATR-dependent checkpoint. Genes Dev 19: 1040-1052.

Coue M, Amariglio F, Maiorano D, Bocquet S, Mechali M. 1998. Evidence for different MCM subcomplexes with differential binding to chromatin in Xenopus. Exp Cell Res 245: 282-289.

Cuvier O, Lutzmann M, Mechali M. 2006. ORC is necessary at the interphase-to-mitosis transition to recruit cdc2 kinase and disassemble RPA foci. Curr Biol 16: 516-523.

Cuvier O, Stanojcic S, Lemaitre JM, Mechali M. 2008. A topoisomerase II-dependent mechanism for resetting replicons at the S-M-phase transition. Genes Dev 22: 860-865.

Delmolino LM, Saha P, Dutta A. 2001. Multiple mechanisms regulate subcellular localization of human CDC6. I Biol Chem 276: 26947-26954.

Evrin C, Clarke P, Zech J, Lurz R, Sun J, Uhle S, Li H, Stillman B, Speck C. 2009. A double-hexameric MCM2-7 complex is loaded onto origin DNA during licensing of eukaryotic DNA replication. Proc Natl Acad Sci 106: 20240-20245.

Fletcher RJ, Bishop BE, Leon RP, Sclafani RA, Ogata CM, Chen XS. 2003. The structure and function of MCM from archaeal M. thermoautotrophicum. Nat Struct Biol 10: 160-167.

Forsburg SL. 2004. Eukaryotic MCM proteins: Beyond replication initiation. Microbiol Mol Biol Rev 68: 109-131.

Hua XH, Yan H, Newport J. 1997. A role for Cdk2 kinase in negatively regulating DNA replication during $S$ phase of the cell cycle. J Cell Biol 137: 183-192.

Hyrien O, Mechali M. 1993. Chromosomal replication initiates and terminates at random sequences but at regular intervals in the ribosomal DNA of Xenopus early embryos. EMBO J 12: 4511-4520.

Ishimi Y. 1997. A DNA helicase activity is associated with an MCM4, -6, and -7 protein complex. J Biol Chem 272: 2450824513.

Kanter DM, Bruck I, Kaplan DL. 2008. Mcm subunits can assemble into two different active unwinding complexes. I Biol Chem 283: 31172-31182. 
Kim DU, Hayles J, Kim D, Wood V, Park HO, Won M, Yoo HS, Duhig T, Nam M, Palmer G, et al. 2010. Analysis of a genome-wide set of gene deletions in the fission yeast Schizosaccharomyces pombe. Nat Biotechnol 28: 617-623.

Lemaitre JM, Bocquet S, Mechali M. 2002. Competence to replicate in the unfertilized egg is conferred by Cdc6 during meiotic maturation. Nature 419: 718-722.

Lemaitre JM, Danis E, Pasero P, Vassetzky Y, Mechali M. 2005. Mitotic remodeling of the replicon and chromosome structure. Cell 123: 787-801.

Lutzmann M, Mechali M. 2008. MCM9 binds Cdt1 and is required for the assembly of prereplication complexes. Mol Cell 31: 190-200.

Lutzmann M, Maiorano D, Mechali M. 2006. A Cdt1-geminin complex licenses chromatin for DNA replication and prevents rereplication during $S$ phase in Xenopus. EMBO $J$ 25: 5764-5774.

Maiorano D, Lemaitre JM, Mechali M. 2000. Stepwise regulated chromatin assembly of MCM2-7 proteins. I Biol Chem 275: 8426-8431.

Maiorano D, Cuvier O, Danis E, Mechali M. 2005. MCM8 is an MCM2-7-related protein that functions as a DNA helicase during replication elongation and not initiation. Cell 120: 315-328.

Matsuyama A, Arai R, Yashiroda Y, Shirai A, Kamata A, Sekido S, Kobayashi Y, Hashimoto A, Hamamoto M, Hiraoka Y, et al. 2006. ORFeome cloning and global analysis of protein localization in the fission yeast Schizosaccharomyces pombe. Nat Biotechnol 24: 841-847.

Mendez J, Zou-Yang XH, Kim SY, Hidaka M, Tansey WP, Stillman B. 2002. Human origin recognition complex large subunit is degraded by ubiquitin-mediated proteolysis after initiation of DNA replication. Mol Cell 9: 481-491.

Mimura S, Takisawa H. 1998. Xenopus Cdc45-dependent loading of DNA polymerase $\alpha$ onto chromatin under the control of S-phase Cdk. EMBO J 17: 5699-5707.

Moore JD, Kornbluth S, Hunt T. 2002. Identification of the nuclear localization signal in Xenopus cyclin E and analysis of its role in replication and mitosis. Mol Biol Cell 13: 43884400.

Nguyen VQ, Co C, Li JJ. 2001. Cyclin-dependent kinases prevent DNA re-replication through multiple mechanisms. Nature 411: 1068-1073.

Prokhorova TA, Blow JJ. 2000. Sequential MCM/P1 subcomplex assembly is required to form a heterohexamer with replication licensing activity. J Biol Chem 275: 2491-2498.

Remus D, Diffley JF. 2009. Eukaryotic DNA replication control: Lock and load, then fire. Curr Opin Cell Biol 21: 771-777.

Remus D, Beuron F, Tolun G, Griffith JD, Morris EP, Diffley JF. 2009. Concerted loading of Mcm2-7 double hexamers around DNA during DNA replication origin licensing. Cell 139: 719-730.

Sakwe AM, Nguyen T, Athanasopoulos V, Shire K, Frappier L. 2007. Identification and characterization of a novel component of the human minichromosome maintenance complex. Mol Cell Biol 27: 3044-3055.

Schwacha A, Bell SP. 2001. Interactions between two catalytically distinct MCM subgroups are essential for coordinated ATP hydrolysis and DNA replication. Mol Cell 8: 1093-1104.

Takahashi N, Lammens T, Boudolf V, Maes S, Yoshizumi T, De Jaeger G, Witters E, Inze D, De Veylder L. 2008. The DNA replication checkpoint aids survival of plants deficient in the novel replisome factor ETG1. EMBO J 27: 1840-1851.

Takahashi N, Quimbaya M, Schubert V, Lammens T, Vandepoele $\mathrm{K}$, Schubert I, Matsui M, Inze D, Berx G, De Veylder L. 2010. The MCM-binding protein ETG1 aids sister chromatid co- hesion required for postreplicative homologous recombination repair. PLoS Genet 6: e1000817. doi: 10.1371/journal. pgen. 1000817.

Walter J, Newport JW. 1997. Regulation of replicon size in Xenopus egg extracts. Science 275: 993-995.

Yanagida M. 2009. Clearing the way for mitosis: Is cohesin a target? Nat Rev Mol Cell Biol 10: 489-496.

Yanow SK, Lygerou Z, Nurse P. 2001. Expression of Cdc18/Cdc6 and Cdt1 during G2 phase induces initiation of DNA replication. EMBO J 20: 4648-4656.

You Z, Ishimi Y, Masai H, Hanaoka F. 2002. Roles of Mcm7 and Mcm4 subunits in the DNA helicase activity of the mouse Mcm4/6/7 complex. J Biol Chem 277: 42471-42479.

Zou L, Elledge SJ. 2003. Sensing DNA damage through ATRIP recognition of RPA-ssDNA complexes. Science 300: 15421548 . 


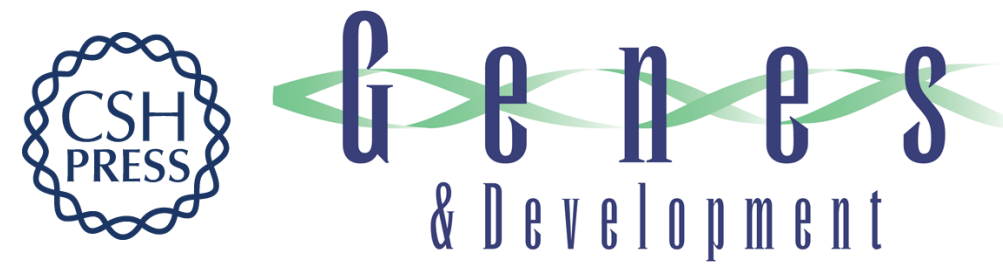

\section{MCM-BP regulates unloading of the MCM2-7 helicase in late S phase}

Atsuya Nishiyama, Lori Frappier and Marcel Méchali

Genes Dev. 2011, 25: originally published online December 31, 2010

Access the most recent version at doi:10.1101/gad.614411

\section{Supplemental \\ Material \\ http://genesdev.cshlp.org/content/suppl/2010/12/20/gad.614411.DC1 \\ References This article cites 40 articles, 21 of which can be accessed free at: http://genesdev.cshlp.org/content/25/2/165.full.html\#ref-list-1 \\ License}

Email Alerting Service

Receive free email alerts when new articles cite this article - sign up in the box at the top right corner of the article or click here.

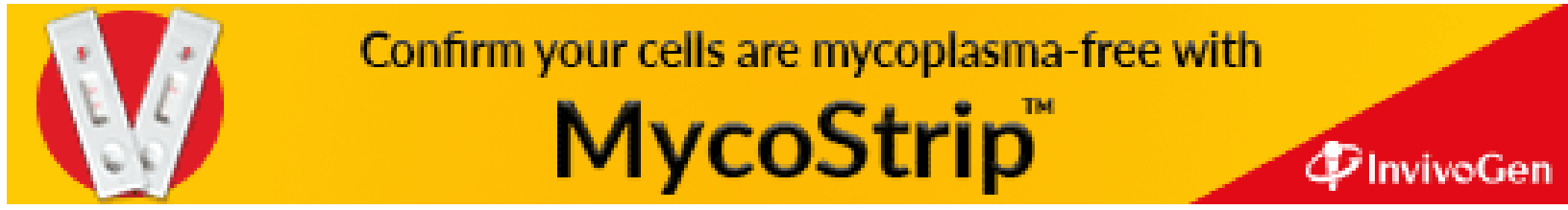

